\title{
Joint Power Allocation and Cell Formation for Energy-Efficient VLC Networks
}

\author{
Mohanad Obeed*, Anas M. Salhab*, Salam A. Zummo*, and Mohamed-Slim Alouini ${ }^{+}$ \\ ${ }^{*}$ King Fahd University of Petroleum \& Minerals, Dhahran, Eastern Province, Saudi Arabia \\ Email: \{g201106250, salhab, zummo\}@kfupm.edu.sa \\ ${ }^{+}$King Abdullah University of Science and Technology, Thuwal, Makkah Province, Saudi Arabia \\ Email: slim.alouini@kaust.edu.sa
}

\begin{abstract}
In this paper, we propose a joint cell formation and power allocation algorithms for energy efficiency (EE) maximization in visible light communication (VLC) networks. Unlike the previous works, we show that the cell formation and power allocation are interlinked problems and they should be solved jointly. We start by proposing a new algorithm for users clustering and then associating all the access points (APs) to the clustered users based on a proposed metric. Under the assumption that the vectored transmission is applied at each formed cell, we solve an optimization problem that aims to maximize the EE by allocating the powers for the users with quality of service $(\mathrm{QoS})$ constraints. Then, we propose an algorithm that jointly allocates the power and decides which APs must participate in communication and which ones must be switched off. The numerical results demonstrate that the proposed algorithms significantly improve the EE compared to the traditional methods and algorithms.
\end{abstract}

Index Terms - Visible light communication, energy efficiency, clustering, power allocation.

\section{INTRODUCTION}

As known, the radio frequency (RF) communications are becoming more restricted because of the limited spectrum resources in wireless networks. As a solution, the VLC has been introduced in indoor environment to overcome the RF limitations and provide better services to the users. The VLC is a communication system that uses light emitting diode (LED), which is highly energy efficient, as a transmitter to emit both the light and information signals to the users. We should note that the power of the information signal must be in the range that the illumination requirement is satisfied and in the range of the LEDs' physical limits. The receiver must be equipped with a photo detector (PD) device that converts the optical signal to an electrical signal. Hence, the data is transmitted using an intensity modulation (IM) technique at the transmitter and received using a direct detection (DD) technique at the receiver (IM/DD). Unlike the RF network, the VLC works properly only when the line of site (LoS) component between the transmitter and receiver is available.

Extensive work has been done for throughput and system capacity maximization in VLC networks using different modulation schemes [1], [2], resource allocation and load balancing [3], [4], and using multi-input multi-output schemes [5]. Despite all the aforementioned advances, few papers in literature addressed the design and optimization issues of the energy efficiency (EE) in VLC networks. However, with the advent of $5 \mathrm{G}$ of wireless networks and the tremendous number of access points (APs) and billions of connected devices, the demand for designing energy-efficient system is becoming even more compelling [6]. Aiming to enhance the EE in VLC, several factors have been employed in literature such as cell formation, power allocation, and link level optimization. Because the inter-cell interference is a major reason behind QoS and EE degradation, the cell formation has been proposed to mitigate this problem by grouping multiple APs to be in a one cell. Cell formation design is a crucial step that has a great impact on the EE of the system. In a highly dense VLC networks, when the number of users is much less than the number of APs, the user-centric (UC) design for VLC networks is an appropriate technique for cell formation. In [7], the authors studied the UC design principle for VLC, where the resultant user-to-network association structure is based on amorphous cell-shapes. They first clustered the users then associated the APs to the grouped users. While in [8], the authors extended the work to enhance the fairness among users. Authors of [9] allocated the powers for the clustered users aiming to maximize the $\mathrm{EE}$ of the distributed cells. All the previous works [8]-[10] studied the system level optimization for energy efficient VLC system by solving the cell formation and power allocation separately, while they are interlinked problems. Finding the global optimal solution for the joint cell formation and power allocation is an extremely difficult problem. However, we propose a suboptimal solution by considering the APs association and power allocation jointly.

In this paper, we design an energy efficient VLC network by proposing a new efficient algorithms that jointly form the cells, allocate the powers for the users, and select the APs. Specifically,

- We propose a new user clustering algorithm that aims to minimize the distance of the clustered users to their centers and maximize the distance between the different centers in order to mitigate the inter-cell interference.

- Unlike the previous works, we establish a metric for each AP in order to select the appropriate user cluster to work on.

- We show that the power allocation and cell formation problems are not separated problems as shown in the previous works. Therefore, we develop an algorithm that solves both problems jointly which leads to a significant 


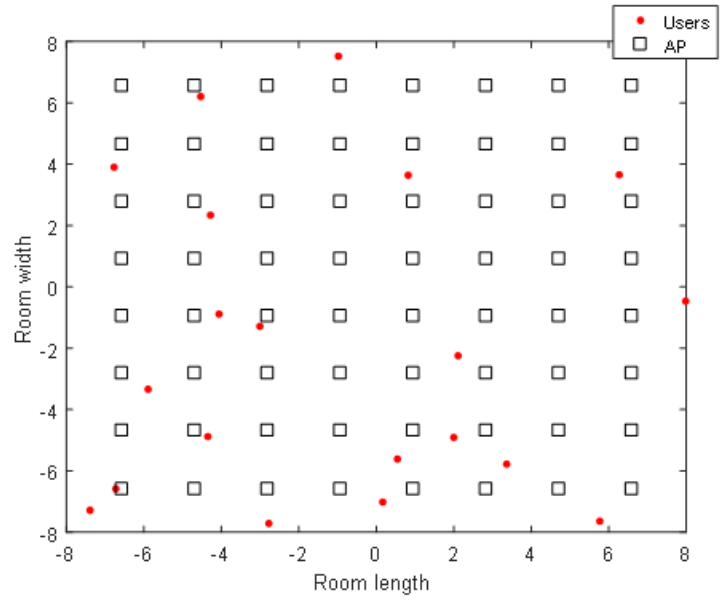

Fig. 1: System model.

improvement in the EE.

- The numerical results show that there exists an optimal number of clusters to achieve the highest EE under a given user clustering and AP association algorithm.

The rest of this paper is organized as follows. The system and channel models are introduced in Section II. In Section III, we present the problem formulation. Some simulation results are presented and discussed in Section IV. Finally, the paper is concluded in Section V.

\section{System AND Channel Models}

The system under consideration consists of $N_{A}$ VLC APs and $N_{u}$ users, as shown in Fig. 1. The users are distributed uniformly in the room and the APs are fixed in the ceiling of the room. Each AP is equipped with multiple LEDs that use IM to transmit the light signal to the users, which receive the light by a PD. Also, the locations of users are assumed to be unchanged during a short time duration $T$. Thus, the channelstate-information (CSI) of the VLC links is considered to be constant during this period. We study this system under the assumption that the number of users is much less than the number of APs.

\section{A. Channel Model}

The LoS VLC channel between the $i^{t h}$ LED and the $j^{t h}$ user can be expressed as follows [11]

$$
h_{i, j}^{(v)}=\frac{(m+1) A_{p}}{2 \pi d_{i, j}^{2}} \cos ^{m}(\phi) g_{o f} f(\theta) \cos (\theta),
$$

where $m$ is the Lambertian index that is given by $m=$ $-1 / \log _{2}\left(\cos \left(\theta_{1 / 2}\right)\right.$, where $\theta_{1 / 2}$ is the half intensity radiation angle, $A_{p}$ is the physical area of the PD, $d_{i, j}$ is the distance between the $i^{t h}$ LED to the $j^{\text {th }}$ user, $g_{o f}$ is the gain of the optical filter, $\phi$ is the angle of radiance at the LED, $\theta$ is the angle of incidence at the PD, and $f(\theta)$ is the optical concentrator gain, which is a function of $\theta$ that is given by $f(\theta)=\frac{n^{2}}{\sin ^{2}(\Theta)}$ if $\theta \leq \Theta$ or 0 if $\theta>\Theta$, where $n$ is the refractive index and $\Theta$ is the semi-angle of the field-of-view (FoV) of the PD. In a VLC network, the LED has to operate in the linear region so that the optical power at its output is a linear function of the input voltage. The non-LoS VLC transmissions are assumed to be unsuccessful so that we work only on the LoS paths. This assumption does not affect the proposed algorithm since it does not depend on a specific channel model. Therefore, when the LoS path is available, $h_{i, j}^{(v)}$ is given by (1); otherwise, $h_{i, j}^{(v)}=0$.

\section{B. Cell Formation}

Here, we provide a new UC clustering algorithm under a given number of clusters, then we provide a new algorithm for APs association to the formed users' clusters. Our target in users clustering is to maximize the separation between the clusters or in other words, maximize the distances between the edge users who belong to different cells. The main contribution of our clustering approach is the initial step that has a significant impact on the final results.

1) User-centric clustering: In this section, we aim to group the users in a predefined $K$ clusters based on their distances to each other. Here, we have two targets, namely having the distances between the cluster centers as far as possible, and having the summation of the distances of all users in a cluster to their center as small as possible. The first target contributes in decreasing the inter-cell interference, while the second target contributes in enhancing the energy efficiency inside the cells when we ignore the inter-cell interference. The traditional K-means clustering method has been proposed to cluster the users based on their distances to each other. It is designed to minimize the following objective function

$$
U=\sum_{k \in K} \sum_{j \in c}\left\|x_{j, k}-c_{k}\right\|^{2},
$$

where $x_{j, k}$ is the position of the user $j$ in the cluster $k, c_{k}$ is the center of the cluster $k$. There is multiple improved versions of $\mathrm{K}$-means method in literature. Here, we adopt the improved version of the K-means method proposed in [12], which is called K-means++. More details on the Kmeans++ clustering method are provided in [12]. The main disadvantage in the $\mathrm{K}$-means++ method is that the selection of the initial centers may lead to a poor clustering since it is based on a probabilistic selection. Therefore, we propose a new algorithm that provides a robust selection for the initial centers that outperforms the K-mean++ in terms of maximizing and improving the EE.

If we have $K$ points and the distances between them are $d_{i, j}, i=1, \ldots, K, j=i+1, \ldots, K$ and the summation of distances between all points is constrained to equal $D$, the solution of the following optimization problem

$$
\max _{d_{i, j}} \prod_{i=1, j=i+1}^{K} d_{i, j}, \text { s.t. } \sum_{i=1, j=i+1}^{K} d_{i, j} \leq D,
$$

is that $d_{i, j}=\frac{D}{K}, i=1, \ldots, K, j=i+1, \ldots, K$. This means that if we have $M K$ centers, the maximum separable $K$ centers out of $M K$ can be found by finding the product of the distances of all possible $K$ centers and pick up the maximum result, where $M$ is an integer value greater than or equal to 1 . The main idea of the proposed algorithm is to start with a number of initial centers that is much more than $K$, then we select the centers that are maximum separable 
using the idea above. Therefore, the following algorithm is the proposed clustering algorithm: Increasing $M$ would

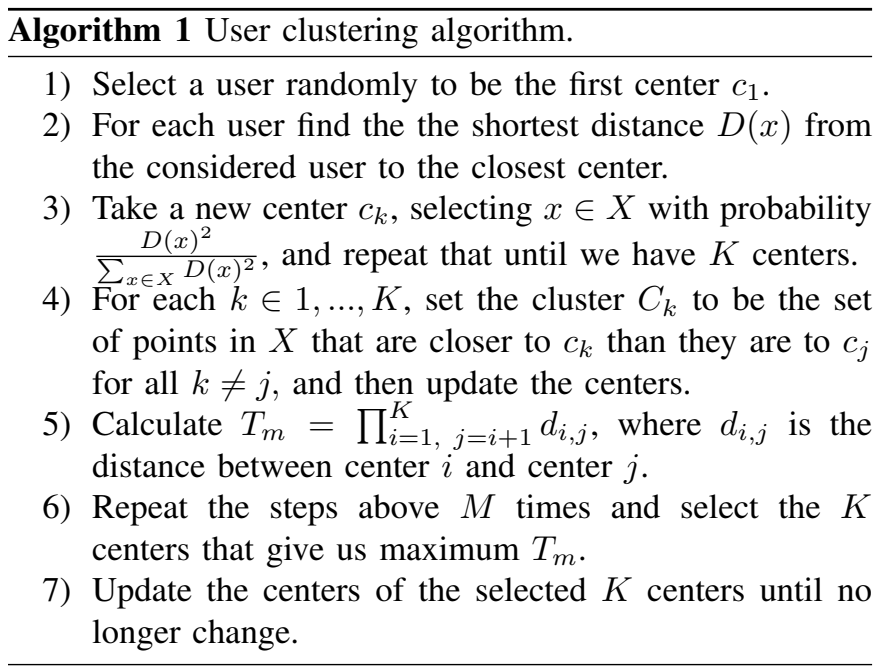

enhance the performance and increase the complexity, and decreasing it would decrease the complexity and degrade the performance.

2) AP Association: It is direct to know that increasing the number of APs in a cluster would enhance the EE of that cluster, but on the other hand, it increases the inter-cell interference in the other clusters. This inspires us to propose a new cluster formation technique that first involves all the APs in the network, then switches off the ones that are harmful (in terms of increasing the inter-cell interference) more than helpful (in terms of enhancing the EE inside the cell). Here, we distribute the APs to the clustered users, and in Section III-B, we optimize the APs selection based on a given power allocation. Our target here is that for each AP, we select the best users cluster that might serve the associated users or enhance the EE as possible as it can. Therefore, we propose two steps to associate the APs to the formed cells:

1) We assign for each user its closest AP by using the following procedures: 1) In the channel matrix, find the maximum channel value, assign the corresponding AP to the corresponding user, then make the row and the column of the corresponding pair equal to zero, 2) Repeat Step (1) until the channel matrix equals the zero matrix.

2) For the remaining APs, for each cluster $c$, we first find the average channel of all APs to all users in the cluster $c$ as follows

$$
\hat{d}_{i, c}=\sum_{j \in c} h_{j, i}^{2} \cdot c=1, . ., K, i=1, . ., N_{A} .
$$

Then, we associate the AP $i$ to the cluster that satisfies this relation

$$
I_{i}=\max _{c}\left(\hat{d}_{i, c}\right), c=1, . ., K .
$$

The target of the second step is to associate the AP to the cluster that would maximize the cell capacity and minimize the inter-cell interference in the other cells.

\section{PROBLEM Formulation}

The problem here is how to allocate the powers for all users with selecting the APs that help in enhancing the EE. After forming the cells, we first solve the power allocation problem, then we provide an algorithm that jointly solves the power allocation problem and APs selection. In each cell, we have $N_{A, c}$ APs transmitting the signal vector $\mathbf{Y}_{t, c} \in \mathbb{R}^{N_{A, c} \times 1}$ to $N_{u, c}$ users, and the received signal vector is given by

$$
\mathbf{Y}_{r, c}=\rho \mathbf{H}_{c} \mathbf{G}_{c} \overline{\mathbf{P}}_{c} \mathbf{Y}_{t, c}+\mathbf{n}_{\mathbf{c}},
$$

where $\mathbf{H}_{c} \in \mathbb{R}^{N_{u, c} \times N_{A, c}}$ is the channel attenuation matrix, $\overline{\mathbf{P}}_{c}=\operatorname{diag}\left(\mathbf{P}_{c}\right)$, where $\mathbf{P}_{c}$ is the electronic power vector assigned to the users belong to the cluster $c, \mathbf{n}_{\mathbf{c}}$ is the noise plus the inter-cell interference, and $\mathbf{G}_{c}$ is the pre-coding matrix that is assumed here to be designed to diagonalize the channel matrix by equating it by $\mathbf{G}_{c}=\mathbf{H}_{\mathbf{c}}{ }^{H}\left(\mathbf{H}_{\mathbf{c}} \mathbf{H}_{\mathbf{c}}{ }^{H}\right)^{-1}$.

\section{A. Power Allocation Scheme}

Our goal in this section is to allocate the power for the $N_{u}$ users to maximize the EE. Specifically, the objective function is to maximize the EE in each formed cell under certain QoS constraints and maximum available power constraints. These constraints are formulated to guarantee some fairness among users per cell and achieve the required illumination, respectively. In literature, two types of EE function have been introduced, which are the average per-cell EE and the global EE. For the average per-cell EE, let us define $\eta_{c}$ as the EE at the cell $c$, hence the average per cell EE of the whole network is defined as

$$
E E=\frac{1}{K} \sum_{c=1}^{K} \eta_{c}
$$

where

$$
\eta_{c}=\frac{\beta \sum_{j=1}^{N_{u, c}} \log \left(1+\frac{\left(\rho^{2} / 2\right) P_{j, c}}{B N_{0}+X_{c, j}}\right)}{\sum_{i=1}^{N_{A, c}} \sum_{j=1}^{N_{u, c}} g_{i, j}^{2} P_{j, c}},
$$

where $P_{j, c}$ is the assigned power for the user $j$ in the cell $c$, $X_{c, j}$ is the interference received at the user $j$ in the cell $c$, and $\beta$ is a value which depends on the applied modulation scheme. Because of the assumption that the (ACO-OFDM) modulation scheme is applied, the value of $\beta=B / 4$, where $B$ is the modulation bandwidth. While the global EE (GEE) is the summation of throughput of all users in all clusters over the total power consumed at all APs, which can be expressed as

$$
G E E=\frac{\beta \sum_{c=1}^{K} \sum_{j=1}^{N_{u, c}} \log \left(1+\frac{\left(\rho^{2} / 2\right) P_{j, c}}{B N_{0}+X_{c, j}}\right)}{\sum_{c=1}^{K} \sum_{i=1}^{N_{A, c}} \sum_{j=1}^{N_{u, c}} g_{i, j}^{2} P_{j, c}} .
$$

Function (8) usually optimized when the centralized approach is employed, while Function (6) supports an efficient distributed approach. Here, we adopt the average per-cell EE as an objective function, where the optimization Problem can be separated equivalently to $K$ subproblems. Therefore, the optimization problem in cluster $c$ can be expressed as follows: 


$$
\begin{array}{cl}
\max _{P_{j, c}, j=1, . ., N_{u, c}} & \eta_{c}, \\
\text { s.t. } & R_{j, c} \geq \gamma, j=1, \ldots, N_{u, c} \\
& \sum_{j=1}^{N_{u, c}} g_{i, j}^{2} P_{j, c} \leq p_{\max }, i=1, . ., N_{A, c}, \\
& P_{j} \geq 0 \quad j=1, . ., N_{u, c} .
\end{array}
$$

where $R_{j, c}=\beta \log \left(1+\frac{\left(\rho^{2} / 2\right) P_{j, c}}{B N_{0}+X_{c, j}}\right)$ is the achievable rate at the user $j$ in the cell $c, \gamma$ is the minimum required data rate, and $p_{\max }$ is the maximum power available at the APs. Problem (9) must be implemented in each cell independently aiming to maximize the summation of the EE in the whole network. Problem (9) is not easy to be solved because the inter-cell interference terms depend on the allocated powers and the power allocation problem depends on the interference terms. In order to simplify this problem, we solve Problem (9) under the worst case where the interference is assumed to be at its maximum value, which happens when all the APs are assumed to transmit at their maximum power. The objective function in (9) is a ratio of two functions (concave in the numerator and a linear function in the denominator), which is generally considered as a non-convex function. It is noted that there is no standard approach for solving non-convex optimization problems. However, this class of optimization problems, which are called fractional programming can be solved using the most common Dinkelbach method [14] that transforms this problem into a successive concave functions. Using Dinkelbach method, we can transform the objective function in (9) to be as

$$
\begin{array}{ll}
\max _{P_{j, c}, j=1, . ., N_{u, c}} & \beta \sum_{j=1}^{N_{u, c}} \log \left(1+\frac{\left(\rho^{2} / 2\right) P_{j, c}}{B N_{0}+X_{c, j}}\right) \\
& -q_{c} \sum_{i=1}^{N_{A, c}} \sum_{j=1}^{N_{u, c}} g_{i, j}^{2} P_{j, c} \\
\text { s.t. } & (9 a),(9 b),(9 c),
\end{array}
$$

where $q_{c}$ is a variable introduced to be optimized or selected to have the above function equal to zero at the optimality. If $q_{c}$ is given, Problem (10) is concave and can be solved using the sub-gradient method [15]. In order to find the optimal $q_{c}$, Dinkelbach algorithm used to find the optimal value of $q_{c}$ by: Step 1) put $q_{c}=0$ as an initial value, Step 2) solve Problem (10) and let $P_{j, c}^{*}$ is the optimal solution, Step 3) update $q_{c}$ by

$$
q_{c}=\frac{\beta \sum_{j=1}^{N_{u, c}} \log \left(1+\frac{\left(\rho^{2} / 2\right) P_{j, c}^{*}}{B N_{0}+X_{c, j}}\right)}{\sum_{i=1}^{N_{A, c}} \sum_{j=1}^{N_{u, c}} g_{i, j}^{2} P_{j, c}^{*}},
$$

Step 4) repeat Step 2 and Step 3 until the objective function of Problem (10) is close to zero value.

\section{B. APs Selection}

In this section, we optimize the APs that must participate in the communication aiming to minimize the intercell interference. It is clear that, under an optimal power allocation strategy, as we increase the participating APs in the transmission, the EE is enhanced as long as the intercell interference is omitted. Hence, first, we classify the APs $\overline{\text { Algorithm } 2 \text { Joint APs selection and power allocation for EE }}$ maximization.

1) Find all interfering APs, and sort them ascendingly based on their $\Upsilon_{i, c}$ value.

2) For $q=1: L$

3) For each interfering AP, starting from the AP that has the lowest $\Upsilon_{i, c}$ value, change its status (if it is on, switch it off and if it is off, switch it on) with keeping the condition that the number of the active APs is greater than the number of users in each cluster satisfied.

4) Implement the power allocation problem and check if the EE is improved. If not, return the AP to its previous status.

5) If $(E E(q-1)-E E(q)<\epsilon)$, break.

6) End for.

into three categories: 1) the APs that have zero channels to all users, 2) the APs that have channels to associated users but zero channels to the users belonging to other cells (i.e. non-interfering APs), 3) the interfering APs that have LoS to their associated users and also have LoS to the users in the other clusters (i.e. interfering APs). The second type of the APs must be switched on because their participation in the communication improves the $\mathrm{EE}$ as long as the power allocation optimization problem is applied. Our interest is in the third type where each one of the APs has to be selected carefully to be switched on or switched off. Participating these APs definitely improves the EE in their own cells, but on the other hand, reduces the EE of the other cells by causing inter-cell interference. Thus, in the following, we establish a metric that approximately measures the eligibility of each AP to participate in the communication (in case it causes non-zero inter-cell interference). This metric of the AP $i$ in cluster $c$ can be given by

$$
\Upsilon_{i, c}=\frac{\sum_{j \in c} h_{j, i}^{2}}{\sum_{j \notin c} h_{j, i}^{2}} . \forall i \forall c .
$$

High value of $\Upsilon_{i, c}$ means that the quality of the information signal coming from the AP $i$ to the intended users is much greater than the emitted interference from that AP and vise versa. It is important to note that the APs selection highly depends on the allocated power since allocating the power for the users is also allocating the transmit powers at the APs. Consequently, the APs selection and the power allocation problems are interlinked, which motivates us to propose an iterative algorithm that finds the joint solution for both of them. The following algorithm solves the power allocation and APs selection alternatively until they converge. The benefit behind sorting the APs ascendingly based on their $\Upsilon_{i, c}$ values is that we guarantee satisfying the condition that the number of active APs is greater than the number of users in each cluster with the APs that have highest $\Upsilon_{i, c}$. In addition, sorting the APs ascendingly increases the rate of convergence significantly. Algorithm 2 is guaranteed to converge because with each change in the AP status in Step 3, the EE either improves or stays as is. 
TABLE I: Simulation Parameters

\begin{tabular}{lc}
\hline Name of the Parameter & Value of the Parameter \\
\hline Room hight & $3 \mathrm{~m}$ \\
Maximum bandwidth of VLC AP, $B$ & $20 \mathrm{MHz}$ \\
The physical area of a PD, $A_{p}$ & $0.1 \mathrm{~cm}^{2}$ \\
Half-intensity radiation angle, $\theta_{1 / 2}$ & $60^{\circ}$ \\
Half FoV (H-FoV) semi-angle of PD, $\Theta$ & $45^{\circ}-55^{\circ}$ \\
Gain of optical filter, $g_{o f}$ & 1 \\
Refractive index, $n$ & 1.5 \\
Efficiency of converting optical to electric, $\rho$ & $0.53[\mathrm{~A} / \mathrm{W}]$ \\
Maximum transmit power, $p_{\max }$ & $0.386 \mathrm{Watt}$ \\
Noise power spectral density of LiFi, $N_{0}$ & $10^{-22} \mathrm{~A}^{2} / \mathrm{Hz}$ \\
\hline
\end{tabular}

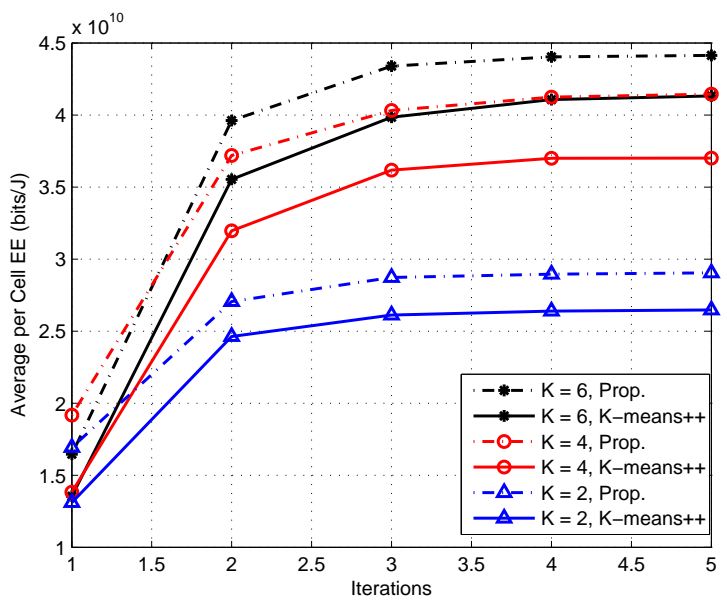

Fig. 2: Energy efficiency versus number of iterations in Algorithm 2 with different number of clusters and different users clustering.

\section{Simulation Results}

In the following, we verify the capability of the proposed algorithms for enhancing the performance of the VLC network. We show the convergence of the proposed algorithms and how they enhance the energy efficiency. A $15 \times 15$ room area is assumed with 64 VLC APs fixed in the ceiling. The values of all used parameters in the considered VLC system are given in Table I. Monte-Carlo simulation is used to asses the performance of the proposed algorithms where in each simulation iteration, a uniform random user distribution is generated. Fig. 2 shows the convergence of Algorithm 2. It is clear that Algorithms 2 needs at most three iterations to converge. Furthermore, the number of iterations needed to converge does not depend on the number of clusters in the system. In addition, it can be seen that only the first iteration yields a significant improvement in the EE of the system, especially when the number of clusters is large. Fig. 2 also shows the superiority of our proposed users clustering algorithm over the K-means++ algorithm.

Fig. 3 illustrates how the EE behaves as we increase the number of cells. Besides, it shows the significant improvement in the EE when we apply Algorithm 2 compared to participating all the APs in the transmission. This figure shows unexpected results since as we know, as the number of cells increases, the inter-cell interference increases which degrades the EE. But, this figure shows that the average per-cell EE increases and then decreases with the number of cells, which

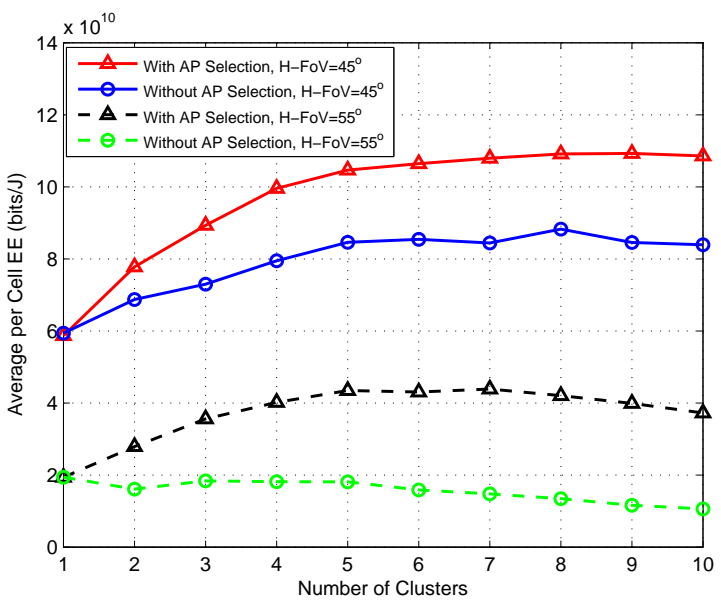

Fig. 3: Comparison between applying Algorithm 2 for AP selection and participating all APs by plotting EE versus number of clusters with different H-FoV.

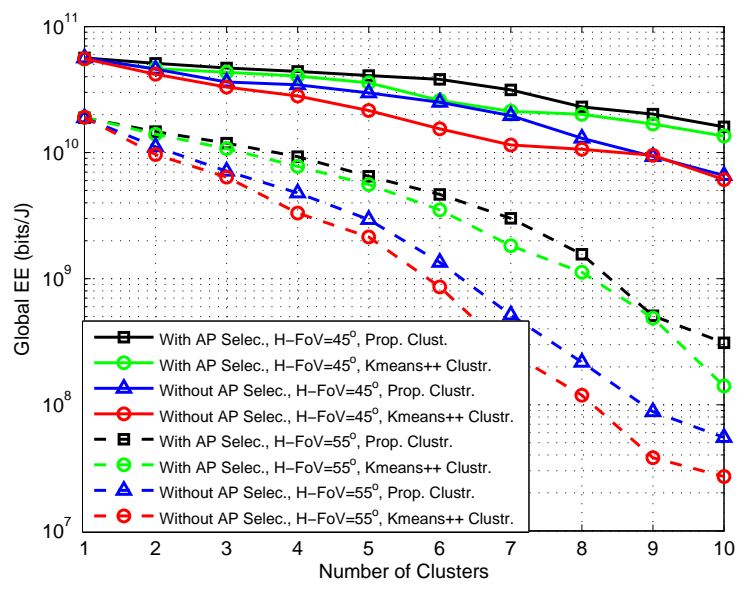

Fig. 4: Comparison between applying Algorithm 2 for AP selection and participating all APs by plotting Global EE versus number of clusters with different $\mathrm{H}-\mathrm{FoV}$.

means that there is another factor that helps in enhancing the average per-cell EE as the number of cells increases. Apparently, this factor has a great impact when the intercell interference is small which occurs when the number of clusters is small or when the H-FoV is small. On the other hand, the global EE is decreasing with increasing the number of cells as shown in Fig. 4, which means that this factor is approximately absent in global EE. The reason behind that is the definition of both the average per-cell EE and the global EE. If we have $x_{1}, x_{2}, y_{1}$, and $y_{2}$ as any positive numbers, it is easy to show that $\frac{x_{1}+x_{2}}{y_{1}+y_{2}}<\frac{x_{1}}{y_{1}}+\frac{x_{2}}{y_{2}}$. This means if we suppose that the interference and the received powers at all users remain fixed with increasing the number of clusters, 


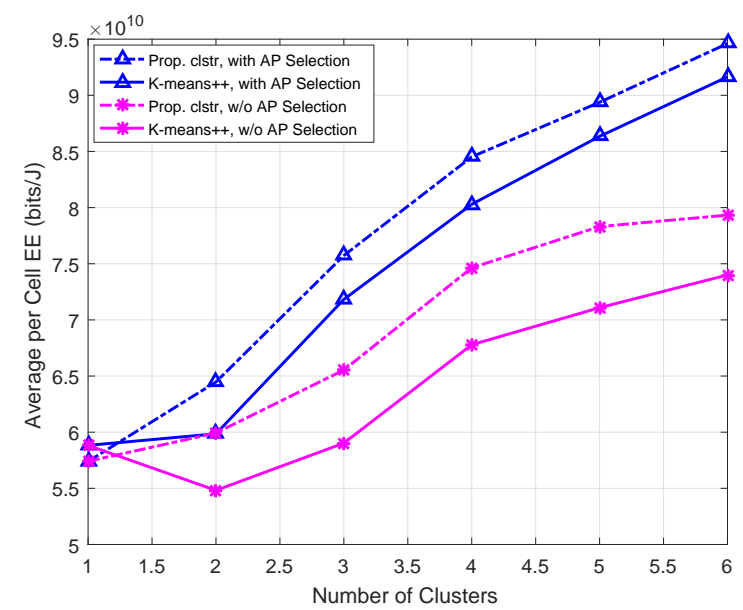

Fig. 5: Comparison between the K-means++ and the proposed clustering methods with and without AP selection by plotting $\mathrm{EE}$ versus number of cells when $\mathrm{H}-\mathrm{FoV}=45^{\circ}$.

mathematically, we obtain that

$$
\begin{aligned}
& \overbrace{\frac{\sum_{c=1}^{K} \sum_{j=1}^{N_{u, c}} R_{j, c}}{\sum_{c=1}^{K} \sum_{j=1}^{N_{A, c}} p_{j, c}}}^{\text {Global EE }}<\overbrace{\frac{\sum_{j=1}^{N_{u, 1}} R_{j, 1}}{\sum_{j=1}^{N_{A, 1}} p_{j, 1}}+\frac{\sum_{j=1}^{N_{u, 2}} R_{j, 2}}{\sum_{j=1}^{N_{A, 2}} p_{j, 2}}}^{K=2} \\
& K=3 \\
& <\overbrace{\frac{\sum_{j=1}^{N_{u, 1}} R_{j, 1}}{\sum_{j=1}^{N_{A, 1}} p_{j, 1}}+\frac{\sum_{j=1}^{N_{u, 2}} R_{j, 2}}{\sum_{j=1}^{N_{A, 2}} p_{j, 2}}+\frac{\sum_{j=1}^{N_{u, 3}} R_{j, 3}}{\sum_{j=1}^{N_{A, 3}} p_{j, 3}}}^{\sum_{j,}} .
\end{aligned}
$$

The inequality above can be extended to $K=N_{u}$. Hence, when the amount of increased interference, as we increase the number of cells, is small, this factor dominates and leads to increase the average per-cell EE with the number of cells. Fig. 4 confirms the above argument by showing that the GEE decreases as we increase the number of clusters. This is because the only factor that affects the GEE as we increase the number of cells is the inter-cell interference. Fig. 4 also shows that the proposed user clustering algorithm outperforms the Kmeans++ algorithm in terms of GEE. Besides, Algorithm 2 provides a significant improvement in GEE as shown in the figure.

Fig. 5, shows the effectiveness of the proposed algorithms in improving the EE of the considered network. The best performance occurs when we apply the AP selection along with the proposed user clustering algorithm, while the worst performance happens when the K-maens++ user clustering algorithm is used with all the APs participating in communication.

\section{CONClusion}

In this paper, an energy efficient visible light communication network was designed based on amorphous cell formation with jointly allocating the power and associating the APs. A new user clustering algorithm was proposed based on distances between users and based on separating the clusters as much as possible to mitigate the inter-cell interference. Another algorithm was proposed to jointly allocate the power and select the APs in order to maximize the EE. The results showed that there exists an optimal number of clusters to achieve the highest average per-cell EE under a given cell formation algorithm.

\section{ACKNOWLEDGMENT}

This work was supported by the Deanship of Scientific Research in King Fahd University of Petroleum \& Minerals through grant number KAUST004. The authors would like also to acknowledge the KFUPM-KAUST research initiative resulted from this research work.

\section{REFERENCES}

[1] A. Yesilkaya, E. Basar, F. Miramirkhani, E. Panayirci, M. Uysal, and H. Haas, "Optical MIMO-OFDM with generalized LED index modulation," IEEE Trans. Commun., vol. 65, no. 8, pp. 2429-3441, 2017.

[2] J. Armstrong, "OFDM for optical communications," IEEE/OSA J. Lightw. Technol., vol. 27, no. 3, pp. 189-204, 2009.

[3] X. Li, R. Zhang, and L. Hanzo, "Cooperative load balancing in hybrid visible light communications and WiFi," IEEE Trans. Commun., vol. 63, no. 4, pp. 1319-1329, 2015.

[4] Y. Wang, D. A. Basnayaka, and H. Haas, "Dynamic load balancing for hybrid Li-Fi and RF indoor networks," in Proc. IEEE Int. Conf. Commun. Workshops (ICCW), London, UK, 2015, pp. 1422-1427.

[5] A. Nuwanpriya, S.-W. Ho, and C. S. Chen, "Indoor MIMO visible light communications: Novel angle diversity receivers for mobile users," IEEE J. Sel. Areas Commun., vol. 33, no. 9, pp. 1780-1792, 2015.

[6] S. Buzzi, I. Chih-Lin, T. E. Klein, H. V. Poor, C. Yang, and A. Zappone, "A survey of energy-efficient techniques for 5G networks and challenges ahead," IEEE J. Sel. Areas Commun., vol. 34, no. 4, pp. 697-709, 2016.

[7] R. Zhang, J. Wang, Z. Wang, Z. Xu, C. Zhao, and L. Hanzo, "Visible light communications in heterogeneous networks: Paving the way for user-centric design," IEEE Wireless Commun., vol. 22, no. 2, pp. 8-16, 2015.

[8] X. Li, F. Jin, R. Zhang, J. Wang, Z. Xu, and L. Hanzo, "Users first: User-centric cluster formation for interference-mitigation in visible-light networks," IEEE Trans. Wireless Commun., vol. 15, no. 1, pp. 39-53, 2016.

[9] R. Zhang, H. Claussen, H. Haas, and L. Hanzo, "Energy efficient visible light communications relying on amorphous cells," IEEE J. Sel. Areas Commun., vol. 34, no. 4, pp. 894-906, 2016.

[10] X. Li, Y. Huo, R. Zhang, and L. Hanzo, "User-centric visible light communications for energy-efficient scalable video streaming," IEEE Trans. Green Commun. Netw., vol. 1, no. 1, pp. 59-73, 2017.

[11] J. M. Kahn and J. R. Barry, "Wireless infrared communications," Proc. IEEE, vol. 85, no. 2, pp. 265-298, 1997.

[12] D. Arthur and S. Vassilvitskii, "K-means++: The advantages of careful seeding," in Proc. The 8th Annual ACM-SIAM Sympo. Discrete Algorithms. Society for Industrial and Applied Mathematics, 2007, pp. $1027-1035$.

[13] M. Obeed, A. M. Salhab, S. A. Zummo, and M.-S. Alouini, "Joint load balancing and power allocation for hybrid VLC/RF networks," in Proc. IEEE Global Commun. Conf. (Globecom), Singapore, 2017.

[14] W. Dinkelbach, "On nonlinear fractional programming," Management Science, vol. 13, no. 7, pp. 492-498, 1967.

[15] S. Boyd and L. Vandenberghe, Convex Optimization. Cambridge University Press, 2004. 